\title{
Oscillation Criteria for Third Order Nonlinear Differential Equations with a Nonpositive Neutral Term
}

\author{
Said R. Grace \\ Cairo University, Department of Engineering Mathematics, Faculty of Engineering, Orman, Giza 12613, Egypt \\ Email: saidgrace@yahoo.com
}

\begin{abstract}
We present new oscillation criteria for a class of third order nonlinear differential equations with a nonpositive neutral term. The results obtained improve and complement some related results known in the literature. Two illustrative examples are provided.
\end{abstract}

Keywords: Oscillation, third order, neutral differential equation, nonpositive neutral term.

\section{Introduction}

This paper is concerned with the oscillatory behavior of solutions of nonlinear third-order differential equations with a nonpositive neutral term of the form

$$
(x(t)-p(t) x(\sigma(t)))^{\prime \prime \prime}+q(t) x^{\beta}(\tau(t))=0, \quad t \geq t_{0}>0,
$$

subject to the following conditions:

(i) $\beta$ is the ratio of positive odd integers;

(ii) $p, q:\left[t_{0}, \infty\right) \rightarrow(0, \infty)$ are continuous functions and $0 \leq p(t) \leq p_{0}<1$;

(iii) $\tau, \sigma:\left[t_{0}, \infty\right) \rightarrow R$ are continuous functions with $\tau(t) \leq t, \sigma(t) \leq t, \tau^{\prime}(t)>0, \sigma^{\prime}(t)>0$, and $\lim _{t \rightarrow \infty} \tau(t)=\lim _{t \rightarrow \infty} \sigma(t)=\infty$.

By a solution of equation (1), we mean a function $x \in C\left(\left[T_{x}, \infty\right), R\right)$ for some $T_{x} \geq t_{0}$ which has the property $x(t)-p(t) x(\sigma(t)) \in C^{3}\left(\left[T_{x}, \infty\right), R\right)$ and satisfies $(1)$ on $\left[T_{x}, \infty\right)$. We consider only those solutions of (1) which satisfy $\sup \{|x(t)|: t \geq T\}>0$ for all $T \geq T_{x}$ and assume that (1) possesses such solutions. A solution $x(t)$ of $(1)$ is said to be oscillatory if it has arbitrarily large zeros, i.e., for any $t_{1} \in\left[t_{0}, \infty\right)$ there exists $t_{2} \geq t_{1}$ such that $x\left(t_{2}\right)=0$; otherwise it is called nonoscillatory, i.e., it is eventually of one sign. Equation (1) is said to be oscillatory if all its solutions oscillate.

In recent years, there has been much research activity concerning the oscillation and nonoscillation of solutions of different classes of differential equations with linear and nonlinear neutral term, and we refer the reader to the papers [1], [2], [3], [4], [5], [6], [7], [10], [11], [13], [14], [15] and the references therein as examples of recent results on this topic. A commonly employed condition is

$$
-1<p(t) \leq 0
$$

as well as the condition

$$
0 \leq p(t)<1
$$

However, oscillatory behavior of solutions of differential equations with a nonpositive neutral term are not very prevalent in the literature; some results can be found in [3], [6] , [7] and [11] for third order equations and [4], [10] and [13] for second order equations. We point out that the sufficient conditions established in these papers ensure that any solution $x(t)$ of the equation considered either oscillates or converges to zero as $t \rightarrow \infty$. This means that these results cannot distinguish solutions with different behaviors.

Here we wish to develop some new sufficient conditions which ensure that every solution of (1) is oscillatory. In this connection, the results in the present paper improve many known results in the relevant literature, and furthermore, can easily be extended to the more general third order differential equations as well as second order differential equations with a nonpositive neutral term. For these reasons, we expect that the results presented in this paper will contribute significantly to study of oscillation of solutions of various classes of differential equations with a nonpositive neutral term. 


\section{Main Results}

We begin with the following new comparison criterion.

Theorem 2.1: Let $h(t)=\sigma^{-1}(\tau(t)) \leq t$ for $t \geq t_{0}$ and assume that there exists an increasing function $\eta:\left[t_{0}, \infty\right) \rightarrow(0, \infty)$ such that $\tau(t) \leq \eta(t) \leq t$ for $t \geq t_{0}$. If the equations

$$
K^{\prime}(t)+\frac{\theta}{2^{\beta}} \tau^{2 \beta}(t) q(t) K^{\beta}(\tau(t))=0
$$

for some constant $\theta \in(0,1)$,

$$
Z^{\prime}(t)+q(t)\left(\frac{(\eta(t)-\tau(t))^{2}}{2}\right)^{\beta} Z^{\beta}(\eta(t))=0
$$

and

$$
W^{\prime}(t)+(\theta h(t))^{\beta}\left(\int_{t}^{\infty} q(s) d s\right) W^{\beta}(h(t))=0,
$$

are oscillatory, then equation (1) is oscillatory.

Proof : Let $x(t)$ be a nonoscillatory solution of (1), say $x(t)>0, x(\tau(t))>0$ and $x(\sigma(t))>0$ for $t \geq t_{1}$ for some $t_{1} \geq t_{0}$.

Let

$$
y(t)=x(t)-p(t) x(\sigma(t)), \quad t \geq t_{0} .
$$

Then, from equation (1) and condition(ii) we see that

$$
y^{\prime \prime \prime}(t)=-q(t) x^{\beta}(\tau(t))<0, \quad t \geq t_{1},
$$

and hence $y^{\prime \prime}(t)$ is decreasing and eventually of one sign. That is, there exists a $t_{2} \geq t_{1}$ such that $y^{\prime \prime}(t)>0$ or $y^{\prime \prime}(t)<0$ for $t \geq t_{2}$. We claim that $y^{\prime \prime}(t)>0$ for $t \geq t_{2}$. To prove the claim, suppose that there exists $t_{2} \in\left[t_{1}, \infty\right)$ such that $y^{\prime \prime}(t)<0$ for $t \geq t_{2}$. Then, in view of (6) there exist a $t_{3} \geq t_{2}$ and a positive constant $\mu$ such that

$$
y^{\prime \prime}(t) \leq y^{\prime \prime}\left(t_{3}\right):=-\mu<0 \text { for } t \geq t_{3} .
$$

Integrating the last inequality twice from $t_{3}$ to $t$, we conclude that $\lim _{t \rightarrow \infty} y(t)=-\infty$.

Now, we consider the following two cases:

Case 1: If $x(t)$ is unbounded, then there exists a sequence $\left\{t_{k}\right\}$ such that $\lim _{k \rightarrow \infty} t_{k}=\infty$ and $\lim _{k \rightarrow \infty} x\left(t_{k}\right)=\infty$, where $x\left(t_{k}\right)=\max \left\{x(s): t_{0} \leq s \leq t_{k}\right\}$. Since $\sigma(t) \rightarrow \infty$ as $t \rightarrow \infty$, for sufficiently large $k$, we have $\sigma\left(t_{k}\right)>t_{0}$. From $\sigma(t) \leq t$, we see that

$$
x\left(\sigma\left(t_{k}\right)\right)=\max \left\{x(s): t_{0} \leq s \leq \sigma\left(t_{k}\right)\right\} \leq \max \left\{x(s): t_{0} \leq s \leq t_{k}\right\}=x\left(t_{k}\right) .
$$

Therefore, for sufficiently large $k$, we obtain

$$
y\left(t_{k}\right)=x\left(t_{k}\right)-p\left(t_{k}\right) x\left(\sigma\left(t_{k}\right)\right) \geq\left(1-p_{0}\right) x\left(t_{k}\right)>0,
$$

which contradicts the fact that $\lim _{t \rightarrow \infty} y(t)=-\infty$.

Case 2: If $x(t)$ is bounded, then $y(t)$ is also bounded, which again contradicts the fact that $\lim _{t \rightarrow \infty} y(t)=$ $-\infty$. This proves the claim and concludes that $y^{\prime \prime}(t)>0$ for $t \geq t_{2}$.

Next, we have two cases to consider (I) $y(t)>0$ for $t \geq t_{2}$ or (II) $y(t)<0$ for $t \geq t_{2}$.

Case (I): Suppose that $y(t)>0$ for $t \geq t_{2}$. Then, from (5) we see that

$$
x(t) \geq y(t) \text { for } t \geq t_{2},
$$

which together with (1) or (6) gives

$$
y^{\prime \prime \prime}(t) \leq-q(t) y^{\beta}(\tau(t))<0 \text { for } t \geq t_{3} \geq t_{2},
$$


and so, by Kiguradze's Lemma (see [8]), we distinguish the following two cases:

(a) $y^{\prime \prime}(t)>0$ and $y^{\prime}(t)>0$ for $t \geq t_{3}$,

(b) $y^{\prime \prime}(t)>0$ and $y^{\prime}(t)<0$ for $t \geq t_{3}$.

Suppose (a) holds. Then, there exist $\theta \in(0,1)$ and a $t_{4} \geq t_{3}$ such that

$$
y^{\prime}(t) \geq \theta t y^{\prime \prime}(t) \quad \text { for } t \geq t_{4} .
$$

Integrating this inequality from $t_{4}$ to $t$ yields

$$
y(t) \geq \frac{\theta}{2} t^{2} y^{\prime \prime}(t), \quad t \geq t_{5}
$$

and hence

$$
y(\tau(t)) \geq \frac{\theta}{2} \tau^{2}(t) y^{\prime \prime}(\tau(t)) \text { for } t \geq t_{6} \geq t_{5},
$$

where we assume $\tau(t) \geq t_{5}$ for $t \geq t_{6}$. Using (8) in (7) gives

$$
K^{\prime}(t)+\frac{\theta}{2^{\beta}} \tau^{2 \beta}(t) q(t) K^{\beta}(\tau(t)) \leq 0,
$$

where $K(t)=y^{\prime \prime}(t)>0$. The function $K(t)$ is obviously strictly decreasing on $\left[t_{3}, \infty\right)$. Hence, by Theorem 2.1 in [12], we conclude that there exists a positive solution $K(t)$ of equation (2) with $\lim _{t \rightarrow \infty} K(t)=0$, which contradicts the fact that equation (2) is oscillatory.

Next, we consider (b). For $v \geq u \geq t_{3}$, one can easily find that

$$
y(u) \geq \frac{(v-u)^{2}}{2} y^{\prime \prime}(v)
$$

Putting $u=\tau(t)$ and $v=\eta(t)$ into (10), we obtain

$$
y(\tau(t)) \geq \frac{(\eta(t)-\tau(t))^{2}}{2} y^{\prime \prime}(\eta(t)) \text { for } t \geq t_{3} .
$$

Using (11) in (7) gives

$$
-y^{\prime \prime \prime}(t) \geq q(t)\left(\frac{(\eta(t)-\tau(t))^{2}}{2}\right)^{\beta}\left(y^{\prime \prime}(\eta(t))\right)^{\beta} .
$$

With $Z(t)=y^{\prime \prime}(t)>0$, the last inequality yields

$$
Z^{\prime}(t)+q(t)\left(\frac{(\eta(t)-\tau(t))^{2}}{2}\right)^{\beta} Z^{\beta}(\eta(t)) \leq 0 .
$$

The rest of the proof is similar to that of Case (a) and hence is omitted.

Case (II). Suppose that $y(t)<0$ for $t \geq t_{2}$. Let $z(t)=-y(t)>0$ for $t \geq t_{2}$. Then, from equation (1), we see that

$$
z^{\prime \prime \prime}(t)=q(t) x^{\beta}\left(\tau(t)>0 \text { for } t \geq t_{2} .\right.
$$

Since $z(t)>0$ and $z^{\prime \prime \prime}(t)>0$, by Kiguradze's Lemma (see [8]), we get $z^{\prime}(t)>0$. From the definition of $z(t)$, and the fact that $y^{\prime \prime}(t)>0$ for $t \geq t_{3}$, we see that $z^{\prime \prime}(t)<0$ for $t \geq t_{3}$. Thus, in view of $z(t)>0$, $z^{\prime}(t)>0$ and $z^{\prime \prime}(t)<0$, there exist $\theta \in(0,1)$ and a $t_{4} \geq t_{3}$ such that

$$
z(t) \geq \theta t z^{\prime}(t) \text { for } t \geq t_{4}
$$

and so

$$
z(h(t)) \geq \theta h(t) z^{\prime}(h(t)), \quad t \geq t_{5} \geq t_{4},
$$

where we assume $h(t) \geq t_{4}$ for $t \geq t_{5}$. From the definition of $y(t)$, we have

$$
z(t)=-y(t)=p(t) x(\sigma(t))-x(t) \leq p(t) x(\sigma(t)),
$$


and so

$$
x(\sigma(t)) \geq z(t) \quad \text { or } \quad x(t) \geq z\left(\sigma^{-1}(t)\right) .
$$

Using this inequality in (13), we obtain

$$
z^{\prime \prime \prime}(t) \geq q(t) z^{\beta}\left(\sigma^{-1}(\tau(t))=q(t) z^{\beta}(h(t)) .\right.
$$

Integrating (15) from $t$ to $u \geq t$ and letting $u \rightarrow \infty$, we get

$$
\begin{aligned}
-z^{\prime \prime}(t) & \geq \int_{t}^{\infty} q(s) z^{\beta}(h(s)) d s \\
& \geq z^{\beta}(h(t)) \int_{t}^{\infty} q(s) d s .
\end{aligned}
$$

Using (14) in (16), we obtain the inequality

$$
-z^{\prime \prime}(t) \geq(\theta h(t))^{\beta}\left(z^{\prime}(h(t))\right)^{\beta} \int_{t}^{\infty} q(s) d s .
$$

With $W(t)=z^{\prime}(t)>0,(17)$ becomes

$$
W^{\prime}(t)+(\theta h(t))^{\beta}\left(\int_{t}^{\infty} q(s) d s\right) W^{\beta}(h(t)) \leq 0 .
$$

The rest of the proof is similar to that of Case (a) and hence is omitted. This completes the proof.

Next, applying the results established in [9] to Theorem 2.1, we get the following corollary.

Corollary 2.1 : Let $\beta=1$. If

$$
\begin{gathered}
\liminf _{t \rightarrow \infty} \int_{\tau(t)}^{t} \tau^{2}(s) q(s) d s>\frac{2}{e}, \\
\liminf _{t \rightarrow \infty} \int_{\eta(t)}^{t}(\eta(s)-\tau(s))^{2} q(s) d s>\frac{2}{e},
\end{gathered}
$$

and

$$
\liminf _{t \rightarrow \infty} \int_{h(t)}^{t} h(s)\left(\int_{s}^{\infty} q(v) d v\right) d s>\frac{1}{e},
$$

where $h(t)$ and $\eta(t)$ are as in Theorem 2.1, then equation (1) is oscillatory.

Corollary 2.2 : Let $\beta<1$. If

$$
\begin{gathered}
\int_{t_{0}}^{\infty} \tau^{2 \beta}(s) q(s) d s=\infty \\
\int_{t_{0}}^{\infty}(\eta(s)-\tau(s))^{2 \beta} q(s) d s=\infty
\end{gathered}
$$

and

$$
\int_{t_{0}}^{\infty}(h(s))^{\beta}\left(\int_{s}^{\infty} q(v) d v\right) d s=\infty,
$$

where $h(t)$ and $\eta(t)$ are as in Theorem 2.1, then equation (1) is oscillatory. The above corollary follows from (9), (12) and (18); we omit its proof.

Next, we present the following interesting result.

Theorem 2.2 : If

$$
\limsup _{t \rightarrow \infty}\left(\tau^{2 \beta}(t) \int_{t}^{\infty} q(s) d s\right)> \begin{cases}2, & \text { if } \beta=1, \\ 0, & \text { if } \beta<1,\end{cases}
$$




$$
\limsup _{t \rightarrow \infty}\left(\int_{\tau(t)}^{t}(\tau(t)-\tau(s))^{2 \beta} q(s) d s\right)> \begin{cases}2, & \text { if } \beta=1 \\ 0, & \text { if } \beta<1\end{cases}
$$

and

$$
\limsup _{t \rightarrow \infty}\left((h(t)(t-h(t)))^{\beta} \int_{t}^{\infty} q(s) d s\right)> \begin{cases}1, & \text { if } \beta=1 \\ 0, & \text { if } \beta<1\end{cases}
$$

where $h(t)$ is as in Theorem 2.1, then equation (1) is oscillatory.

Proof : Let $x(t)$ be a nonoscillatory solution of $(1)$, say $x(t)>0, x(\tau(t))>0$ and $x(\sigma(t))>0$ for $t \geq t_{1}$ for some $t_{1} \geq t_{0}$. As in the proof of Theorem 2.1, we again have two cases to consider (I) $y(t)>0$ or (II) $y(t)<0$ for $t \geq t_{2}$. Suppose first Case (I) holds. Proceeding as in the proof of Theorem 2.1, we see that (7) holds, and so we have two cases (a) or (b) to consider.

Suppose that (a) holds. Then, we again arrive at (8). Integrating (7) from $t$ to $u \geq t$ and letting $u \rightarrow \infty$, we get

$$
y^{\prime \prime}(t) \geq\left(\int_{t}^{\infty} q(s) d s\right) y^{\beta}(\tau(t))
$$

Using (8) in the above inequality, we obtain

$$
\begin{aligned}
y^{\prime \prime}(t) & \geq\left(\int_{t}^{\infty} q(s) d s\right)\left(\frac{\theta}{2} \tau^{2}(t) y^{\prime \prime}(\tau(t))\right)^{\beta} \\
& \geq\left(\int_{t}^{\infty} q(s) d s\right)\left(\frac{\theta}{2} \tau^{2}(t)\right)^{\beta}\left(y^{\prime \prime}(t)\right)^{\beta},
\end{aligned}
$$

which can be written as

$$
\left(y^{\prime \prime}(t)\right)^{1-\beta} \geq\left(\int_{t}^{\infty} q(s) d s\right)\left(\frac{\theta}{2} \tau^{2}(t)\right)^{\beta} .
$$

Taking the limsup as $t \rightarrow \infty$ in the last inequality, we obtain a contradiction to (19).

Next, we consider case (b). Then, (10) holds for $v \geq u \geq t_{3}$. For $t \geq s \geq t_{3}$, putting $u=\tau(s)$ and $v=\tau(t)$ into (10), we obtain

$$
y(\tau(s)) \geq \frac{(\tau(t)-\tau(s))^{2}}{2} y^{\prime \prime}(\tau(t)) .
$$

Integrating (7) from $\tau(t)$ to $t$ and using (22), we see that

$$
\left(y^{\prime \prime}(\tau(t))\right)^{1-\beta} \geq \int_{\tau(t)}^{t} q(s)\left[\frac{(\tau(t)-\tau(s))^{2}}{2}\right]^{\beta} d s .
$$

Taking the limsup as $t \rightarrow \infty$ in the last inequality, we obtain a contradiction to (20).

Next, assume that Case (II) holds. Then, as in the proof of Theorem 2.1, we see that (13), (14) and (16) hold. Next, for $t \geq s \geq t_{3}$, one can easily see that

$$
z^{\prime}(s) \geq(t-s)\left(-z^{\prime \prime}(t)\right)
$$

and so

$$
z^{\prime}(h(t)) \geq(t-h(t))\left(-z^{\prime \prime}(t)\right) \text { for } t \geq t_{4} .
$$

Substituting (23) into (14) gives

$$
z(h(t)) \geq \theta h(t)(t-h(t))\left(-z^{\prime \prime}(t)\right) \text { for } t \geq t_{5} .
$$

Using this inequality in (16), we obtain

$$
\left(-z^{\prime \prime}(t)\right)^{1-\beta} \geq\left(\int_{t}^{\infty} q(s) d s\right) \theta^{\beta}(h(t)(t-h(t)))^{\beta} .
$$


Taking the limsup as $t \rightarrow \infty$ in the last inequality, we obtain a contradiction to (21). This completes the proof.

Example 2.1 : Consider the nonlinear differential equation with nonpositive neutral term

$$
\left(x(t)-\frac{1}{5} x(t / 2)\right)^{\prime \prime \prime}+\frac{1}{t^{3 / 2}} x^{1 / 3}(t / 3)=0, \quad t \geq 1 .
$$

Here we have $p(t)=1 / 5, \sigma(t)=t / 2, q(t)=1 / t^{3 / 2}, \beta=1 / 3$, and $\tau(t)=t / 3$. Then, $\sigma^{-1}(t)=2 t$ and $h(t)=2 t / 3$. Next, with $\eta(t)=t / 2$, we obtain

$$
\begin{gathered}
\int_{t_{0}}^{\infty} \tau^{2 \beta}(s) q(s) d s=\frac{1}{3^{2 / 3}} \int_{1}^{\infty} \frac{d s}{s^{5 / 6}}=\infty, \\
\int_{t_{0}}^{\infty}(\eta(s)-\tau(s))^{2 \beta} q(s) d s=\frac{1}{6^{2 / 3}} \int_{1}^{\infty} \frac{d s}{s^{5 / 6}}=\infty,
\end{gathered}
$$

and

$$
\int_{t_{0}}^{\infty}(h(s))^{\beta}\left(\int_{s}^{\infty} q(v) d v\right) d s=\left(\frac{16}{3}\right)^{1 / 3} \int_{1}^{\infty} \frac{d s}{s^{1 / 6}}=\infty .
$$

Thus, all conditions of Corollary 2.2 are satisfied and hence equation (24) is oscillatory.

Example 2.2 : Consider the linear differential equation with nonpositive neutral term

$$
\left(x(t)-\frac{t}{2 t+1} x(t / 2)\right)^{\prime \prime \prime}+\frac{k}{t^{3}} x(t / 3)=0, \quad t \geq 1 .
$$

Here we have $p(t)=t /(2 t+1), \sigma(t)=t / 2, q(t)=k / t^{3}$ with $k>36, \beta=1$, and $\tau(t)=t / 3$. Then, $1 / 3 \leq p(t) \leq 1 / 2, \sigma^{-1}(t)=2 t$ and $h(t)=2 t / 3$. As in the Example 2.1, it is easy to verify that all conditions of Theorem 2.2 hold, and so equation (25) is oscillatory by Theorem 2.2 .

Remark : The results of this paper can easily be extended to higher order equations of the form

$$
\left(\left(a(t)(x(t)-p(t) x(\sigma(t)))^{(n-1)}\right)^{\gamma}\right)^{\prime}+q(t) x^{\beta}(\tau(t))=0, n \text { is a positive integer, }
$$

$a, p, q \in C\left(\left[t_{0}, \infty\right), R^{+}\right), \gamma$ and $\beta$ are the ratios of positive odd integers, and $\sigma(t)$ and $\tau(t)$ are defined as in this paper.

\section{References}

1. Agarwal R.P., Bohner M., Li T., Zhang C., A new approach in the study of oscillatory behavior of even-order neutral delay differential equations, Appl. Math. Comput., 2013, 255, 787-794.

2. Agarwal R.P., Bohner M., Li T., Zhang C., Oscillation of second-order differential equations with a sublinear neutral term, Carpathian J. Math., 2014, 30, 1-6.

3. Baculikova B., Dzurina J., Oscillation of third-order neutral differential equations, Math. Comput. Modelling, 2010, 52, 215-226.

4. Dong J.G., Oscillation behavior of second order nonlinear neutral differential equations with deviating arguments, Comput. Math. Appl., 2010, 59, 3710-3717.

5. Doslá Z., Liska P., Oscillation of third-order nonlinear neutral differential equations, Appl. Math. Lett., 2016, $56,42-48$.

6. Dzurina J., Thandapani E., Tamilvanan S., Oscillation of solutions to third-order half-linear neutral differential equations, Electron. J. Differential Equations, 2012, 2012(29), 1-9.

7. Grace S.R., Graef J.R., El-Beltagy M.A., On the oscillation of third order neutral delay dynamic equations on time scales, Comput. Math. Appl., 2012, 63, 775-782.

8. Kiguradze I.T., On the oscillation of solutions of equation $d^{m} u / d t^{m}+a(t)|u|^{m}$ sgnu $=0$, Mat.Sb., 1964, 65, $172-187$.

9. Koplatadze R.G., Chanturiya T.A., Oscillating and monotone solutions of first-order differential equations with deviating argument (in Russian), Differ. Uravn., 1982, 18, 1463-1465.

10. Li Q., Wang R., Chen F., Li T., Oscillation of second-order nonlinear delay differential equations with nonpositive neutral coefficients, Adv.Differ. Equ., 2015, 2015:35, 1-7. 
11. Mihalíková B., Kostiková E., Boundedness and oscillation of third order neutral differential equations, Tatra Mt. Math. Publ., 2009, 43, 137-144.

12. Philos C.G., On the existence of nonoscillatory solutions tending to zero at $\infty$ for differential equations with positive delays, Arch. Math., 1981, 36, 168-178.

13. Qin H., Shang N., Lu Y., A note on oscillation criteria of second order nonlinear neutral delay differential equations, Comput. Math. Appl., 2008, 56, 2987-2992.

14. Yang Q., Yang L., Zhu S., Interval criteria for oscillation of second-order nonlinear neutral differential equations, Comput. Math. Appl., 2003, 46, 903-918.

15. Wong J.S.W., Necessary and sufficient conditions for oscillation of second order neutral differential equations, J. Math.Anal. Appl., 2000, 252, 342-352. 